\title{
NUEVAS CONDICIONES JUVENILES Y TRAYECTORIAS DE VIDA EN CUBA: UNA APROXIMACIÓN AL TEMA
}

\author{
LUIS GÓMEZ SUÁREZ* \\ MARÍA JOSEFA LUIS LUIS**
}

\begin{abstract}
RESUMEN
Abordamos aquí un tema escasamente estudiado en el país y que en la actualidad posee una considerable trascendencia para comprender a nuestras juventudes. En el trabajo se valoran las posibles trayectorias de vida de los jóvenes cubanos en su paso desde el ámbito de la formación escolar organizada al del trabajo, es decir a la plena inserción sociolaboral y sus posibles variables. En otros términos se trata de examinar cómo los estudiantes devienen trabajadores, lo que supone determinar los posibles itinerarios que estos describen para insertarse en el mundo adulto. El interés por dicho asunto estriba en la creciente diversificación de las trayectorias juveniles a la vida adulta en las condiciones de un país como Cuba donde se desarrolla un complejo proceso de transición al socialismo.
\end{abstract}

\section{PALABRAS CLAVE: TRANSICIÓN, ITINERARIOS JUVENILES, INTEGRACIÓN SOCIOLABORAL}

* Máster en Historia por la Universidad de La Habana. Investigador Agregado del Centro de Estudios Sobre la Juventud y profesor de la Universidad de La Habana. Especialista en temas del movimiento juvenil y políticas de juventud. E-Mail: ces@jovenclub.cu.

** Máster en Sociología por la Universidad de La Habana. Investigadora Auxiliar del Centro de Estudios Sobre la Juventud y profesora de Historia de la Universidad de La Habana. Especialista en temas relacionados con la inserción laboral de la juventud. E-Mail: ces@jovenclub.cu. 


\title{
NOVAS CONDIÇÕES JUVENIS E TRAJETÓRIAS DE VIDA EM CUBA: UMA APROXIMAÇÃO AO TEMA
}

\begin{abstract}
RESUMO
Abordamos aqui um tema pouco estudado no país e que atualmente é de considerável importância para a compreensão de nossas juventudes. Neste artigo avaliam-se as possíveis trajetórias de vida de jovens cubanos em seus percursos da formação escolar ao mundo do trabalho, ou seja, à plena inserção sociotrabalhista e suas possíveis variáveis. Em outras palavras, trata-se de examinar como os estudantes se tornam trabalhadores, determinando os possíveis itinerários que eles descrevem para inserir-se no mundo adulto. $\mathrm{O}$ interesse por tal tema inscreve-se na crescente diversificação das trajetórias juvenis rumo à vida adulta nas condições de um país como Cuba, onde ocorre um processo complexo de transição ao socialismo.
\end{abstract}

PALAVRAS-CHAVE: TRANSIÇÃO, ITINERÁRIOS JUVENIS,
INTEGRAÇÃO SOCIOTRABALHISTA

NEW YOUTH AND PATH CONDITIONS OF LIFE IN CUBA: AN

APPROACH TO THE SUBJECT

\begin{abstract}
We approach a subject rarely studied here in the country and which now has considerable significance for understanding our youth. At work are valued potential life trajectories of young Cubans on their way from the field of school education organized at work, namely the complete integration and its potential socio variables. In other words it is examining how students becomes workers, which involves identifying the possible routes that they describe in entering the adult world. The interest in this matter lies in the growing diversity of youth trajectories to adulthood under conditions of a country like Cuba where it develops a complex process of transition to socialism.
\end{abstract}

KEY WORDS: TRANSITION, YOUTH ITINERARY, INTEGRATION AND LABOR 


\section{NUEVAS CONDICIONES JUVENILES Y TRAYECTORIAS DE VIDA ¿DE QUÉ SE TRATA?}

EL TEMA DE LAS nuevas condiciones juveniles y trayectorias de vida en Cuba no ha gozado de la suficiente atención de las ciencias sociales, sin embargo, dada la diversificación de las formas de transición abiertas en el país tras las reformas económicas de los años 90, se hace necesario su estudio a fin de favorecer la integración social de las nuevas generaciones en las complejas circunstancias por las que se atraviesa.

En principio, es preciso esclarecer que cuando nos referimos a las condiciones juveniles, estamos llamando la atención acerca de las transformaciones acaecidas en el mundo y en Cuba en las últimas décadas con el advenimiento de la sociedad informacional, también conocida como del conocimiento en los países desarrollados, las cuales están influyendo en los modos de vida de las personas, con los consiguientes cambios en el funcionamiento de la sociedad. Estos cambios, de incuestionable repercusión en el orden socioeconómico y cultural, modifican la estructura social y los modos de asumir a los jóvenes y a las teorías en torno a ellos, en particular los conceptos de juventud como usualmente se hacía.

Las transformaciones originadas en el ciclo vital de las personas por los procesos mencionados, han dado lugar a modificaciones en el modelo tradicional de organización de la existencia humana en cuanto a tres momentos fundamentales: formación, actividad y jubilación. Esto conduce al replanteamiento de la condición juvenil en el nuevo contexto que vive el país. Para algunos investigadores latinoamericanos la condición juvenil se asume «como un conjunto de cambios a nivel de las vivencias y relacionamientos de los jóvenes en un nuevo escenario social, que trae consigo ciertos elementos de 'nuevas condiciones juveniles', diferenciándolas de la 'situación social de los jóvenes'» (Dávila, 2008:56).

Tal percepción sugiere valorar «cómo los jóvenes viven y experimentan su condición de jóvenes, en un espacio y un tiempo determinado» (Dávila, 2008:56). La nueva noción de juventud se asocia a la dilatación de procesos fundamentales que determinan su contenido: la prolongación como fase de la vida producto de una mayor permanencia en el sistema educativo, la demora de su inserción sociolaboral, el retraso de la emancipación de la familia de origen y de la autonomía residencial, así como de la creación de una familia propia. 
La trayectoria de vida describe las transformaciones acaecidas en los procesos de ingreso a la vida adulta, las que conducen a pensar la juventud como una etapa de transición, abordada por Ericsson (1974) como una moratoria generalmente aceptada, es decir, un tiempo de espera durante el cual el individuo se prepara para asumir roles adultos (Ericsson, 1974). La moratoria se establece a partir de una supuesta regularidad de la trayectoria juvenil y de su homogeneidad social, visión que ha llegado hasta el presente, y que comienza a ser cuestionada. Esta consideración tiende a omitir las diferencias existentes entre los jóvenes por concepto de género, etnia, cultura, poción económica y lugar de residencia, entre otras.

El sociólogo argentino Mario Margulis esclarece la noción de moratoria social, la que considera como el «plazo concedido a cierta clase de jóvenes que les permite gozar de una menor exigencia mientras completan su instrucción y alcanzan su madurez social y económica. Es un período de permisividad, una especie de estado de gracia, una etapa de relativa indulgencia, en que no les son aplicadas con todo su rigor las presiones y exigencias que pesan sobre las personas adultas» (Margulis, 2001:43). Tal proceso, por su universalidad, ha dejado de ser una concesión para constituirse en necesidad del desarrollo humano y social que atraviesa todas las clases, géneros, razas y credos.

Por otro lado, en el presente se abren paso enfoques que nos hablan de transiciones de nuevo tipo a nivel de los sujetos y de las estructuras sociales. En este orden cobra particular relevancia el paso desde el mundo de la formación al mundo del trabajo, entendido como la plena inserción sociolaboral y sus variables asociadas.

La noción de trayectorias alude al tránsito de la dependencia a la emancipación o autonomía social, proceso que se ha modificado por la prolongación en el tiempo de la vida estudiantil y el retraso tanto de la inserción laboral, como de las autonomías de emancipación social de los jóvenes. La transición, al tiempo que transcurre de la infancia a la adultez integrando la biografía del sujeto, es un proceso de reproducción social, donde las trayectorias de los jóvenes son un reflejo de las estructuras y los procesos sociales. Comprende la configuración y percepción de la individualidad y la subjetividad, y las relaciones que se establecen entre los sujetos y las estructuras sociales en las que se desarrollan las subjetividades. En la transición a la vida adulta, el presente de los jóvenes es conformado por la interacción de las experiencias acumuladas en el pasado y de las aspiraciones y los planes para el futuro (Dávila, 2008:57). 
Para las trayectorias juveniles, y para su estudio, es preciso tomar en consideración el grupo social de procedencia, el nivel educacional alcanzado, el tipo de institución escolar en que se cursaron los estudios, el título alcanzado y el tipo de trabajo a que se puede acceder con ese título, y la valoración social y simbólica del título obtenido. Bordieu explica que estas trayectorias pueden ser exitosas o fallidas, en dependencia de las situaciones biográficas de los jóvenes en las que influyen casi de modo determinante los desempeños y credenciales educativas alcanzadas durante el tránsito. A ello se suman el acopio, apropiación y transmisión diferenciada de los capitales cultural, económico, social y simbólico (Dávila, 2008:58).

En la actualidad, los cambios operados en el mundo, resultado de los procesos asociados a la globalización neoliberal, la integración del mercado mundial, el crecimiento demográfico, la declinación de la economía doméstica, el desarrollo tecnológico, la industrialización, la escolarización, los medios masivos de comunicación e información y la elevada urbanización, han modificado la transición de la etapa juvenil a la vida adulta. Las trayectorias de vida de las personas han perdido su tradicional estabilidad secuencial de la existencia. Hoy se ha fracturado el orden lineal de la vida caracterizada por el estudio, la formación laboral, la inserción en el trabajo, el matrimonio y la tenencia de hijos. Muchos de estos eventos no siguen un orden preestablecido o no suceden, adoptando el tránsito un carácter imprevisible, vulnerable y de considerable incertidumbre con diversidad de finales debido a la pluralidad de juventudes y de condiciones juveniles.

En resumen, la transición implica un proceso natural por el que pasa toda persona en cualquier época histórica. La juventud es una de las etapas del ciclo vital, y en dicha etapa, la transición se refiere en lo fundamental, a la adquisición de habilidades, capacidades y derechos considerados como propios de la vida adulta. Es por ello que obtener una elevada cultura, formación profesional y capacidad para la participación y las relaciones sociales cobra tanta importancia en la actualidad para llevar a cabo sus proyectos de vida. La trayectoria se relaciona con el posicionamiento de los sujetos en la estructura social, de acuerdo con las relaciones de poder entre las distintas clases y grupos sociales. Lo señalado entre estos dos conceptos en la actualidad es que la transición a la vida adulta, es decir, los modelos tradicionales generalizados de llegar al mundo de los adultos, han sido sustituidos por trayectorias no lineales que conforman entre los jóvenes proyectos individuales de vida (biografias) heterogéneos, determinando, en alguna medida, su ingreso a la vida adulta. 


\section{LAS NUEVAS CONDICIONES JUVENILES EN CUBA}

En cuanto a Cuba, consideramos que también se han desarrollado nuevas condiciones juveniles que marcan su transición a la vida adulta. Cabría preguntarse y tratar de constatar en alguna medida, si en nuestro medio concurren las situaciones nuevas presentes en otros contextos y que cuestionan el concepto tradicional de moratoria psicosocial como período de preparación para asumir la vida como adultos. Téngase en cuenta que el país no escapa a los procesos que se operan a nivel mundial, al tiempo que se han modificado ostensiblemente las condiciones de interacción social, las formas de concebir las relaciones sociales y las posibilidades de participación social. Aunque Cuba no ha sufrido las secuelas del neoliberalismo, los cambios acaecidos desde la crisis de los 90 han venido produciendo efectos semejantes entre su juventud. Tales procesos constituyen objeto de estudio en diversas instituciones científicas de Cuba. En tal sentido, el análisis realizado por María Isabel Domínguez y María Elena Ferrer acerca de los «efectos del período especial sobre los jóvenes», califica este sector social como el más afectado en los órdenes objetivo (empleo, calificación y movilidad social) y subjetivo (expectativas y valores) por los procesos que se desencadenaron tras la desconexión de Cuba del antiguo campo socialista; criterio que se corrobora en las tres encuestas nacionales sobre juventud realizadas en 1994, 1999 y 2004 por el Centro de Estudios Sobre la Juventud.

Entre los procesos que más han impactado en la población en general y en la juventud en particular, en conjunción con la agudización del bloqueo norteamericano - que genera una cultura del asedio con efectos psicológicos negativos- se encuentran la reconversión y reforma de la economía mediante la apertura al turismo internacional y la introducción de mecanismos de mercado. Todo ello ha repercutido de diverso modo entre la juventud con manifestaciones tales como:

- Incremento de las desigualdades sociales y la fragmentación social.

- Énfasis en la necesidad de realización personal, sin que se aprecie la debida correspondencia entre las formas de realización de necesidades individuales y necesidades sociales.

- Se abre paso una cultura del efecto inmediato.

- Cierta indiferencia por la política.

- Aparición de nuevos códigos de interacción entre adultos y jóvenes, padres e hijos, hombres y mujeres. 
- Desarrollo de nuevas formas de participación-comunicación (religiosa e informal, entre otras).

- Incremento de la interacción con los medios electrónicos, la computación y las autopistas de la información: computadoras, internet, videojuegos, telefonía celular. Si bien son posibilidades que la inmensa mayoría de los jóvenes no poseen en sus hogares, una buena parte de ellos han tenido contacto con tales sistemas en las instituciones docentes y comienzan a influir poderosamente en la subjetividad, generando expectativas e identidades. Esta es una vía que, además, sirve de entrada a los discursos ajenos al proyecto socialista y los valores y principios que defiende, de los cuales se apropian determinados grupos de jóvenes.

La juventud cubana actual creció en el contexto del Período Especial, ${ }^{1}$ con una economía segmentada, para algunos ambigua; éste ha sido su referente. Al contrario de sus mayores, no ha podido establecer comparaciones directas entre las dos últimas épocas de nuestra historia nacional, el capitalismo y el socialismo; sus pautas de conducta se han configurado conforme al sistema que surgió a raíz de las reformas económicas durante los años 1993-1996.

Cuando han arribado a la edad de asumir la política y de participar en ella, lo han hecho en un contexto complejo, que propicia un estado de opinión crítico, situándola en un plano inferior al trabajo, el estudio y el entretenimiento y la diversión; lo cual guarda cierta relación con una tendencia que se abre paso imperceptiblemente de culto al presente, a lo momentáneo. Muchos jóvenes esperan del Estado servicios, derechos y protección, pero sin dar nada a cambio al país, mostrándose críticos con las dificultades que afronta ante la imposibilidad de una solución inmediata.

En la práctica los jóvenes cubanos no escapan a procesos que se manifiestan en otros contextos, sólo que lo hacen de forma peculiar dada nuestras condiciones socioeconómicas, políticas y culturales. La concepción de la Revolución acerca de los jóvenes nunca acogió la irresponsabilidad y la improductividad. Tampoco se les ha negado la posibilidad de ser sujetos sociales. En ocasiones se les ha catalogado como inmaduros, irresponsables y problemáticos —manifestaciones

1 Se considera Período Especial en Cuba a la etapa que se inicia tras el desmantelamiento del socialismo en Europa Oriental. la que se caracteriza por una profunda crisis económica y el esfuerzo realizado por el pueblo para salvar la Revolución y las conquistas del socialismo. 
aún no erradicadas del todo - sin embargo, se ha tratado de buscar su compromiso y la ruptura de una visión peyorativa, que en algunos casos se relaciona con una forma de estigmatización política.

Los elementos que pueden modificar la moratoria son, entre otros, los siguientes:

- La construcción de la adultez es cada vez menos lineal. Muchas familias no están interesadas en que sus hijos trabajen, al menos en entidades estatales.

- Ha disminuido el dominio directo de la familia y el sistema escolar sobre el entorno.

- Se posterga el matrimonio o se abstienen de tener hijos, se prolongan los estudios por cursos de diverso propósito (postgrado, recalificación o actualización).

- En la actualidad, por el redimensionamiento económico y el desarrollo científico-técnico, los adultos están en muchas ocasiones en las mismas condiciones que los jóvenes en cuanto a la necesidad de preparación profesional y para la vida.

- Al parecer, en una parte de la juventud se ha producido la ruptura de secuencias que hasta hace relativamente poco tiempo distinguían el ciclo vital, desapareciendo la tradicional temporalidad en la trayectoria de la vida.

- En la cultura juvenil entra en contradicción la alta velocidad que la distingue con la lentitud de los planes escolares y su pertinencia curricular.

- El énfasis en lo visual, afectivo y sensorial debilita el poder socializador tradicional.

- Entre los jóvenes cubanos existe hoy una mayor conciencia de sus identidades juveniles, las que tienden a reconformarse durante la trayectoria vital.

- La prolongación de la vida y de las condiciones de salud alargan la juventud como estado vital.

- Se está produciendo un inicio temprano de las relaciones sexuales.

- Uniones conyugales provisionales.

- Nuestra juventud posee una elevada escolarización y un nivel escolar promedio de enseñanza media.

- La edad superior, por el contrario, parece extenderse por prolongación de estudios, recalificación profesional, y dependencia económica y habitacional de la familia de origen.

- Resquebrajamiento de la cultura del trabajo y el salario.

- Preeminencia de la música y la danza por encima de otras manifestaciones artísticas. 
- Se realizan prácticas culturales alternativas a las que se desarrollan en los espacios formales.

- Cierta presencia de fenómenos como la prostitución, la drogodependencia y la agresividad.

- Formación en una cultura de la emergencia y el asedio por el bloqueo económico, comercial y financiero al que es sometido el país por los gobiernos norteamericanos.

Si bien es necesario profundizar en los problemas enunciados, por el carácter hipotético que estas consideraciones poseen, de acuerdo con lo explicado, al parecer los jóvenes cubanos no escapan de los procesos que modifican la moratoria que les concede la sociedad.

\section{TRAYECTORIAS DE VIDA DE LOS JÓVENES CUBANOS}

Realicemos, ahora una aproximación a las posibles trayectorias juveniles en nuestro medio. Para el estudio de este asunto se emplean diversos modelos de análisis o tipologías: se puede apelar a los grados de escolaridad alcanzados como principal variable de estudio (Du Bois Reymond et al., 2002), o el paso del estudio al trabajo para la adquisición de la plena autonomía (Casal, en Dávila et al. 2002:62-63). ${ }^{2}$ Ambas presentan aristas interesantes y en gran medida complementarias, toman en consideración los niveles de estudio alcanzados por los jóvenes, lo cual se aviene a las condiciones de nuestro país. Con todo, más que guiarnos por una experiencia metodológica específica, trataremos de exponer la realidad cubana, con sus lógicas especificidades.

Para analizar el caso cubano no es posible soslayar las diferencias socioeconómicas, las proyecciones y realizaciones, así como las prioridades que se establecen en materia de justicia, equidad e igualdad de oportunidades. Si bien es cierto que los jóvenes confrontan limitaciones objetivas para satisfacer determinadas necesidades materiales y sus crecientes expectativas de consumo, así como para acceder a una vivienda propia, lo que limita considerablemente la independencia de la familia de origen; la realización de los proyectos de vida se facilitan gracias al apoyo familiar en los más disímiles ámbitos y a las políticas de juventud que genera el Estado con el propósito de asegurar educación y empleo, mediante numerosos servicios y oportunidades de superación e inserción laboral.

2 Casal parte del paso del estudio al trabajo para la adquisición de la plena autonomía con seis tipos de procesos de transición a la vida adulta aplicado en España. 
No obstante, no podemos obviar el incremento paulatino de proyectos y estrategias familiares e individuales. Los valores sociales de la juventud, sus percepciones, expectativas y estrategias de construcción de proyectos de vida exitosos, han sufrido cambios, lo que propicia que el componente de gestión personal sea cada vez más importante para su consecución. Pese a ello, en el país predominan la razón social y los proyectos colectivos de ascenso sobre el logro personal.

En Cuba, todos los niños y jóvenes tienen acceso al sistema nacional de educación, pese a que en algunas instituciones escolares pueden existir condicionantes para el ingreso, y por consiguiente, excluir y crear desiguales oportunidades entre los jóvenes. En este caso se encuentran las Escuelas Vocacionales de Ciencias Exactas, las Escuelas Deportivas y las Escuelas de Arte, tanto las de nivel medio como superior, pues exigen requisitos especiales de aptitud. En este orden, desempeña un papel considerable la influencia de la familia en la preparación del joven para continuar estudios superiores.

El paso de la enseñanza universitaria tradicional basada en grandes centros nacionales, a la municipalización o universalización de la educación superior, ${ }^{3}$ persiguió eliminar condicionantes para el ingreso a la formación profesional antes vedada para muchos jóvenes, lo que representó un considerable esfuerzo democratizador. Pese a ello, la práctica demostró la conveniencia de la selección por medio de los exámenes de ingreso como vía necesaria para elevar la calidad de la docencia y de sus egresados. A excepción de esta limitante intelectual, el acceso a la educación es universal. De existir agentes de exclusión para el desarrollo de trayectorias de éxito, a nuestro juicio, estos factores son de índole cultural, familiar y personal.

Otra cuestión a tomar en consideración es la referida al empleo. En Cuba la fuerza de trabajo no constituye una mercancía y, por lo tanto, no está sujeta a las condicionantes del mercado, es decir, el trabajo no se rige por la oferta y la demanda y las titularidades más competitivas no son definitorias. La promoción o el ofrecimiento de trabajo no se basa en la competitividad, a pesar de que desde mediados de los años 90 se han incrementado las exigencias en términos de títulos,

3 La universalización de la educación superior es la extensión de la universidad y sus procesos sustantivos a toda la sociedad a través de su presencia en los territorios, permitiendo alcanzar mayores niveles de equidad y de justicia social en la obtención de una elevada cultura integral de los ciudadanos. 
experiencia e idoneidad para acceder a determinados sectores de la economía como el turismo y las telecomunicaciones, entre otros.

Para Joaquín Casal la transición «no es sólo el tránsito de la escuela al trabajo, sino, más bien, un proceso complejo desde la adolescencia social hacia la emancipación plena, a la vida adulta: un proceso que incluye la formación escolar y sus trayectorias dentro de la «escuela de masas», la formación en contextos no formales e informales, las experiencias prelaborales, la transición profesional plena y los procesos de autonomía familiar. Desde esta perspectiva, entonces, la «transición» viene definida como un sistema de dispositivos institucionales y procesos biográficos de socialización que de forma articulada entre sí intervienen en la vida de las personas desde que asumen la pubertad, y que son conductores hacia la adquisición de posiciones sociales que proyectan al sujeto joven hacia la consecución de la emancipación profesional, familiar y social» (Casal, 1996:298).

Se infiere entonces que la socialización laboral previa - esa que se recibe en el medio familiar, escolar y en otros espacios- y la propia inserción laboral, son determinantes en la transición de la juventud a la adultez. De cualquier manera, el asunto resulta polémico, toda vez que, además de los múltiples factores que intervienen en el proceso de transición, los problemas de los jóvenes con relación al trabajo son cada vez más graves. Téngase en cuenta que el desempleo ha devenido en un fenómeno estructural propio de la sociedad capitalista acrecentado por el desarrollo de las nuevas tecnologías, en tanto el grupo social más afectado por sus diferentes manifestaciones son justamente los jóvenes.

En el caso cubano las condiciones que inciden en la trayectoria, como la educación general y profesional están aseguradas en términos generales a todos los niveles del sistema nacional de educación, al igual que el empleo, estos son presupuestos de partida que distinguen la posible tipología de trayectoria de la juventud al mundo adulto. ¿Quiere esto decir que todo está resuelto en este orden? En principio estas ventajas atraviesan por múltiples dificultades e influencias que no hacen idílica la transición en nuestro medio. Esta representa, a nuestro juicio, uno de los rasgos fundamentales de la transición en Cuba. De cualquier manera, en la propuesta de Casal encontramos algunos puntos de coincidencia, por lo que pudiera constituir un referente teórico interesante.

La preparación para la transición desde la escuela tiene una extraordinaria importancia. En realidad está concebido que el sistema 
educacional prepare a los niños y adolescentes para favorecer la inserción laboral en la etapa juvenil. Están demostrados los logros alcanzados en cuanto al nivel de instrucción adquirido, la formación técnica y profesional, las actividades prácticas, entre otras; aunque son aspectos perfectibles en términos de pertinencia y calidad. Dada la forma en que está diseñado el sistema educacional cubano, la enseñanza politécnica constituye la principal vía para el acceso de los jóvenes al trabajo; no obstante, existen otras fuentes de procedencia como los preuniversitarios o las escuelas especializadas de deportes, artes, o las que sostienen las propias empresas y ministerios en las que se prepara el personal necesario.

Un fenómeno interesante, es el número notable de quienes tienden a postergar su inserción laboral para continuar estudios universitarios, comenzando a trabajar al concluirlos. Con el surgimiento de los nuevos Programas de la Revolución, ${ }^{4}$ en la primera década del presente milenio se incorporaron nuevas oportunidades de preparación para insertarse laboralmente; por lo general, en estos casos la inclusión se produce en la fase final de la adolescencia o juventud temprana, proceso que se combina con la continuidad de estudios universitarios gracias a la universalización de la Educación Superior. Por otra parte, el estímulo a proseguir los estudios superiores de mayor nivel, sobre todo maestrías y doctorados, ha contribuido a incrementar la tendencia a aplazar la asunción de otras responsabilidades relacionadas con la pareja y la maternidad.

4 La gradual recuperación de la economía permitió llevar a cabo a partir del año 2000, una transformación social que se expresa en un conjunto de programas sociales (más de 150), a los que el liderazgo político dio el nombre de Programas de la Revolución, que abarcaron importantes espacios de la realidad nacional y esferas decisivas para la existencia y desarrollo de los jóvenes. Los Programas conforman una Política de Juventud que puede ser definida por su carácter y contenido como solidaria, con las miras puestas en la integración social de los sectores juveniles que, como resultado de la crisis y las políticas destinadas a solventarla, han quedado desvinculados de los servicios de educación y empleo, presentan situaciones de riesgo, han cometido cualquier tipo de delito o padecen alguna discapacidad. Se parte de la concepción de que los afectados pueden ser rescatados para la sociedad la que está, por principio, obligada a ello. Los programas se dirigen también a los sectores de la población en situaciones de desventaja como personas de la tercera edad, las madres solteras $\mathrm{y}$ las personas con necesidades educativas especiales. 
Sin embargo, existen debilidades en la etapa educacional con negativas implicaciones para el proceso de socialización laboral, nos referimos a los problemas de formación vocacional y orientación profesional, el paternalismo escolar y familiar, el formalismo y el carácter reproductivo que en múltiples ocasiones está presente en el proceso de enseñanza-aprendizaje. La insuficiente preparación en algunas especialidades limitan la independencia del joven y su desarrollo como un individuo participativo, le impiden asumir determinados contenidos de su entorno laboral, generando a su vez incomprensiones y rechazo al trabajo.

A nivel organizacional no siempre se crean las condiciones adecuadas para que el joven se inserte en un medio laboral favorable. La carencia de una cultura del trabajo y la insuficiente orientación e información para la ubicación, a veces provocan una equivocada selección, lo que se traduce en problemas de adaptación a la vida laboral. Ello no posibilita un vínculo afectivo positivo con su labor, sobre todo si no es atendido y adiestrado de manera diferenciada, lo que conduce en múltiples ocasiones a la fluctuación o el abandono del puesto de trabajo. Tal situación es posible que se produzca con jóvenes con diferentes niveles de formación y en cualquier espacio económico.

De acuerdo con lo que Casal denomina trayectoria de éxito precoz, en Cuba encontramos jóvenes con altas expectativas de carrera profesional, con prolongación de la formación académica y resultados positivos, en su defecto la opción de una transición profesional susceptible de mejoras graduales a partir de la formación continúa y/o la promoción interna rápida. Esta trayectoria se da en sectores como el de la ciencia, la biotecnología, las telecomunicaciones, las tecnologías de punta, la gestión empresarial y estatal, etc. El país concede elevada prioridad a la inserción laboral de la reserva profesional y científica asegurando su continua superación y recalificación.

Según estudios realizados, como tendencia, estos sujetos proceden de familias de intelectuales y profesionales que influyen en sus hijos para que continúen el derrotero de sus progenitores. Los análisis más recientes aseveran que aún prevalece la tendencia a la reproducción endógena de la intelectualidad, así como la feminización de las universidades. El papel de la mujer en el orden profesional ha ido creciendo de manera considerable durante el período revolucionario. Sin embargo, esta trayectoria no es privativa de la intelectualidad, la igualdad de oportunidades posibilita que también se dé entre familias de obreros y técnicos, interesados en que sus hijos hagan estudios 
superiores a fin de asegurar su futuro. Pese a las diferentes coyunturas por las que ha atravesado el país en los últimos veinte años, la adquisición de un capital cultural y educacional ha continuado siendo uno de los objetivos claves de la familia a alcanzar por sus hijos.

En este período las profesiones de nivel superior se han visto erosionadas por la precaria situación económica del país, lo que se ha dado en catalogar como de pirámide profesional invertida, pues personas que poseen baja escolaridad pueden llegar a obtener ingresos y reconocimiento social por la elevada demanda social de la profesión que desempeñan, por lo general del tipo de obrero calificado como plomeros, albañiles, zapateros y muchas otras; mientras los profesionales reciben salarios relativamente bajos y su reconocimiento social se ha ido deteriorando. No obstante, los profesionales universitarios pueden llegar a poseer ingresos atractivos, a veces gracias a otras actividades complementarias o segundos contratos de trabajo o publicaciones y servicios ocasionales.

Las llamadas trayectorias obreras corresponden a jóvenes orientados hacia la cultura del trabajo manual con escasa calificación básica y profesional. Tales objetivos presuponen un horizonte social limitado, sin grandes aspiraciones intelectuales. Esta trayectoria es una de las más extendidas entre la juventud cubana y se manifiesta entre obreros calificados y no calificados. De alguna manera se asocia a algunas labores del trabajo por cuenta propia. El proyecto social cubano ha privilegiado este tipo de trayectorias por constituir la base de la clase trabajadora, principal fuerza de trabajo en la industria y los servicios. Se ha promovido la formación laboral en ciertos oficios sumamente escasos y de interés para la población, relacionados con la construcción y el mantenimiento a las viviendas, la reparación de efectos electrodomésticos y bienes de uso personal, entre otros. Estas actividades se vieron afectadas ante la demanda de la formación técnica media y superior. Desde finales de los 80 se graduó una suma considerable de técnicos y profesionales en especialidades que no se correspondían con las necesidades del desarrollo del país.

La trayectoria de adscripción familiar, responde a la presencia de empresas o explotaciones familiares, que en Cuba se pueden asociar al trabajo por cuenta propia de personas a las que se suman los hijos como los pequeños agricultores, talleres, artesanías, pequeños restaurantes, etc. Este tipo de trayectoria, que es generada por una situación coyuntural, es una de las más ventajosas, pues ofrecen importantes ingresos que abren las puertas a mayores niveles de vida. No obstante, es una trayec- 
toria más bien escasa, debido a la inversión inicial que en ocasiones requiere y muchos no poseen, y que además, es muy controlada por el Estado, pues se presta para el desvío de recursos y la especulación con artículos deficitarios en las condiciones económicas de Cuba.

Las trayectorias de precariedad se manifiestan entre jóvenes en situaciones intermitentes de desempleo, no originadas por la carencia de fuentes de empleo, sino por falta de interés para trabajar o algún tipo de inconformidad con la ocupación que se posee, lo que provoca una continua rotación laboral en busca de un empleo supuestamente más atractivo, hasta encontrar el trabajo deseado, o por cualquier otra razón extralaboral que conduce a cierta inestabilidad profesional.

Las denominadas trayectorias desestructuradas de la propuesta analítica de Joaquín Casal acerca de los procesos de inserción social y profesional de los jóvenes, en particular en lo concerniente a las distintas modalidades de transición a la vida adulta, pueden contribuir al análisis del complejo fenómeno de los jóvenes desvinculados del estudio y el trabajo en Cuba. En ella se identifican a jóvenes con bajas expectativas de posicionamiento social y el bloqueo sistemático ante la inserción laboral. La mayor parte de la actividad se desarrolla en la economía marginal o en formas de economía sumergida. Parte de las trayectorias en desestructuración generan una tendencia hacia el aislamiento social o hacia la toma de actitudes de acomodación y tensión social, agresividad social y marginalidad. Transcurrir en estas trayectorias implica perder las aptitudes y actitudes para la inserción laboral regular, y su prolongación acelera su desintegración social, rechazo a la cultura del trabajo, pérdida del sentido de responsabilidad, etc. El joven tiende a la situación de exclusión social.

La desvinculación laboral de los jóvenes no es un fenómeno exclusivo del llamado Período Especial, pero este le confiere una connotación particular. El sistema educacional también afectado por la crisis, además de carecer de recursos para el equipamiento de los talleres, laboratorios y libros de texto, debilitó su papel en la formación de valores, la socialización y el control social. Se redujeron las posibilidades de satisfacer las aspiraciones profesionales de los jóvenes; se produjo una brecha importante entre los programas de formación de fuerza de trabajo calificada y las posibilidades reales de la economía para absorberla; ello obligó a la reducción de matrícula en determinadas especialidades del nivel superior y a la reorientación de muchos jóvenes que se estaban formando en la enseñanza técnica profesional por falta de garantía para la ubicación laboral. 
Por otra parte, la preparación para transitar de la escuela al primer empleo es insuficiente. Durante este crucial período los adolescentes cubanos no cuentan con toda la preparación necesaria para formar y descubrir su vocación, tampoco se les ofrece suficiente información y orientación acerca de las posibilidades y oportunidades de estudio que tienen para satisfacer sus aspiraciones laborales. Las decisiones erradas respecto a la continuidad de estudios al concluir la Secundaria Básica se reflejan después en el abandono de estos o en la preparación para una especialidad técnica que luego no desean ejercer. En los últimos años se han elevado los niveles de escolaridad exigidos en los calificadores de cargo, para ponerlos a tono con los altos índices educacionales que posee Cuba. Esto que constituye un logro importante del país, se convierte en problema para la reinserción social y la realización de sus proyectos de vida de la mayoría de los desvinculados, ya que al no alcanzar esos niveles, se produce un distanciamiento entre el nivel de escolaridad alcanzado, sus aspiraciones laborales y las exigencias que se presentan para acceder a esos puestos de trabajo en la actualidad. Esa distancia tiende a profundizarse con el paso del tiempo, porque las expectativas que estos jóvenes poseen son altas y las exigencias de competencias laborales cada vez son más elevadas.

El grado de escolaridad de los padres pudiera estar influyendo en la manera en que se comporta la preparación de estos jóvenes. La mayor proporción de jóvenes con niveles más bajos coincide con aquellos cuya madre y/o padre tienen nivel primario o secundario. En la medida que el nivel escolar de los padres es superior, también lo es para los jóvenes. Si como tendencia hay coincidencia entre el nivel escolar de los jóvenes desvinculados y el de sus padres, con cierta incidencia de padres negros y mestizos entre los de más bajo nivel de escolaridad, aparece entonces como un proceso de reproducción de incuestionable trascendencia social.

El proceso recuperativo que comienza a experimentar la economía durante el segundo lustro de los años 90 hizo retroceder paulatinamente los índices de desocupación, no obstante, la tasa de desempleo juvenil seguía siendo superior a la media. Por otra parte, la propia devaluación del trabajo y los salarios propició el abandono voluntario del empleo, provocando un crecimiento de los inactivos no interesados en estudiar ni trabajar. A pesar de ciertas similitudes en las trayectorias que sigue la juventud desvinculada del estudio y el trabajo hacia la vida adulta, este grupo social no es homogéneo. De tal forma, se puede identificar jóvenes que se encontraban realizando gestiones para 
trabajar, otros en tareas del hogar, los que no realizan ninguna actividad de estudio o trabajo y un grupo nada despreciable que se auto emplean en diversos espacios informales.

Lo que más llama la atención en ese grupo es su diversidad en cuanto a condiciones de vida; por una parte, se distinguen jóvenes con cierta solvencia económica, por contar con fuentes de ingreso estables, procedentes de la manutención familiar, remesas, o la vinculación al trabajo informal con relativa estabilidad; incluso en no pocos casos su nivel de vida está por encima de la media nacional, lo que va acompañado casi siempre de otro modo de pensar y comportamiento social. Otros jóvenes se encuentran en franca desventaja económica, algunos en situaciones extremas y en sus expresiones revelan cierta tendencia al aislamiento social, muy pobre o nula participación social, muy bajas expectativas y una parte se relacionan con la economía sumergida.

La transición a la vida adulta de este segmento juvenil difiere sustancialmente de los procesos de inserción social y profesional en que se involucra la mayoría de los jóvenes cubanos, por cuanto asumen trayectorias que conducen a la desintegración social, toda vez que tienden al aislamiento, al acomodamiento, a la pérdida del sentido de responsabilidad, a rechazar el trabajo y la inserción laboral regular y a vincularse con el mercado negro, el delito y la marginalidad.

Como hemos podido apreciar, en Cuba, al igual que en otros países, se abre paso a la tendencia de los jóvenes a prolongar su condición juvenil posponiendo su independencia y la formación de familia independiente. Muchos permanecen en el hogar paterno por dificultades para mantenerse mediante una economía propia, o debido, entre otros factores, a la imposibilidad de solventar sus necesidades con los ingresos por concepto de trabajo. Los estudios realizados en los últimos años sitúan la manutención familiar como una de las principales vías utilizadas por los jóvenes cubanos -incluyendo a los trabajadores- para satisfacer sus necesidades, de donde se infiere una pérdida de autonomía relativa respecto al núcleo familiar de origen, particularmente en el caso de las mujeres jóvenes.

Durante el último decenio, el Estado y la sociedad cubana en su conjunto han hecho un considerable esfuerzo por restablecer los procesos de integración social de las nuevas generaciones con apreciables oportunidades de superación e inserción laboral, pese a las serias dificultades que afronta el país desde el punto de vista económico. En tal sentido apuntamos las dificultades para encontrar empleo atractivo en términos de ingresos y la imposibilidad de acceder a una vivienda. 
También habría que señalar las perspectivas y alargamiento de los plazos para la creación de sus propias familias o el escaso interés en crearlas, extendiéndose las uniones consensuales de escasa duración y estabilidad. En línea con estas últimas consideraciones se encuentran la baja fecundidad de las mujeres en la edad reproductiva, en no pocos casos asociada a la prioridad otorgada a sus intereses profesionales, tanto de formación como de realización en el ejercicio de la profesión, lo que trae aparejado el envejecimiento de la población con bajos índices de crecimiento e incluso decrecimiento.

Como ocurre en otros contextos, las transformaciones señaladas pueden estar apuntando hacia la existencia entre los jóvenes cubanos de cambios socioculturales con otras formas de asumir y concebir la condición juvenil, con nuevas visiones, subjetividades y modos de vida que desarrollan maneras de vivir el mundo juvenil, diferentes a las distintivas de las generaciones anteriores.

LA HABANA (CUBA), SEPTIEMBRE 2009

RECIBIDO: SEPTIEMBRE 2009

ACEPTADO: OCTUBRE 2009

\section{REFERENCIAS BIBLIOGRÁFICAS}

Álvarez, Elena y Jorge MátTar (2004) (coordinadores): Política social y reformas estructurales: Cuba a principios del siglo XXI. México: CEPAL, PNUD e INIE.

BALARDINI, SERGIO (2003): «La juventud en el siglo XXI». Jóvenes a la vista. Santiago: Interjoven.

CASAL, JOAQUín (1996): «Modos emergentes de transición a la vida adulta en el umbral del siglo XXI. Aproximación sucesiva, precariedad y desestructuración». Revista Española de Investigaciones Sociológicas №75. Madrid: CIS.

DÁvila, OsCAR; FeliPe GHIARdo y CARlos Medrano (2008): Los desheredados. Trayectorias de vida y nuevas condiciones juveniles. Valparaíso: Ediciones CIDPA (cuarta edición).

Du BoIs REYMOND, MANUELA et al. (2002): «Transiciones modernizadas y políticas de desventaja: Países Bajos, Portugal, Irlanda y jóvenes inmigrantes en Alemania». Revista de Estudios de Juventud $\mathrm{N}^{\circ} 56$. Madrid: INJUVE.

ERICSSON, ERIC (1974): Identidad, juventud y crisis. Buenos Aires: Paidós.

GÓMEZ SUÁREZ, LUIS (2004): «Los programas de la Revolución y la política cubana de juventud, 2000-2004». La Habana: CESJ (inédito). 
LUIS LUIS, MARÍA JOSEFA et al. (2008): «La dirección y el trabajo de los cuadros en la inserción y estabilidad laboral de los jóvenes. Informe de investigación». La Habana: CESJ.

MARGULIS, MARIO (2001): «Juventud: una aproximación conceptual». En SOlum Donas BURAK (compilador): Adolescencia y juventud en América Latina. Cartago: Libro Universitario Regional.

Oficina Nacional De Estadísticas (ONE) (2008): Encuesta Nacional de Ocupación. La Habana: Centro de Estudios de Población y Desarrollo.

PÉREZ ISLAS, JosÉ ANTONIO et al. (2008) (coordinadores): Teorías sobre la juventud. Las miradas de los clásicos. México: UNAM. 